\title{
RASTREAMENTO SOROLÓGICO PARA DOENÇAS INFECCIOSAS EM BANCO DE SANGUE COMO INDICADOR DE MORBIDADE POPULACIONAL
}

\author{
Ana Lúcia S. S. de Andrade* \\ Celina Maria Turchi Martelli* \\ Edmo Dias Pinheiro** \\ Cesar Leite Santana*** \\ Francisco Pereira Borges**** \\ Fábio Zicker*
}

\begin{abstract}
ANDRADE, A.L.S.S. de et al. Rastreamento sorologico para doenças infecciosas em banco de sangue como indicador de morbidade populacional. Rev. Saúde públ., S. Paulo, 23:20-5, 1989.

RESUMO: E discutido o valor do rastreamento sorológico para doença de Chagas, sífilis, hepatite B e AIDS realizado por bancos de sangue como indicador de morbidade populacional. Foram analisados os dados referentes a 62.814 doaçðes de sangue obtidas em dois bancos de sangue públicos e três privados correspondendo ao total das doaçðes no período de outubro de 1985 a outubro de 1987 em Goiânia, Goiás (Brasil). A soroprevalência foi comparada com dados obtidos pela notificação compulsória das doenças e com inquéritos epidemiológicos disponiveis. Foi encontrada soroprevalência para AIDS de $0,0 @ \%$ para um único exame de ELISA, estimando-se em 1.900 o número de indivíduos supostamente infectados em Goiás, número compativel com o esperado quando se trabalha com dados de notificação. Para a doença de Chagas, hepatite B e sífilis foram observadas soroprevalências de até 3,3\%, 1,3\% e $4,1 \%$, respectivamente. Foram discutidas as dificuldades encontradas para validação desses resultados pela ausência de notificaçåo compulsória e características particulares dos inquéritos sorológicos.
\end{abstract} sangue.

DESCRITORES: Bancos de sangue. Exames de massa. Sorodiagnostico. Morbidade. Doadores de

\section{INTRODUÇÃO}

Dados sobre morbidade populacional são obtidos usualmente atraves dos sistemas rotineiros de registro de morbidade, da notificação compulsória, de inquéritos especiais ou, eventualmente, pelo rastreamento sorológico em bancos de sangue.

O sistema de informação de morbidade pela notificação compulsória e registros oficiais no Brasil são precários e nem sempre representam a situação epidemiológica real ${ }^{25}$. Os inquéritos epidemiológicos são geralmente dirigidos a grupos populacionais selecionados ${ }^{3}$, em programas especificos ou em condições de pesquisa, refletindo desse modo situaçס̄es particulares de local e tempo, o que dificulta a comparação entre diferentes estudos, a generalização dos resultados e a análise da tendência secular das doenças na população $0^{4,15,18}$.
O rastreamento ou "screening" como método para a detecção de infecção ou doença tem sido amplamente usado nos países mais desenvolvidos em programas de saúde pública, especialmente na detecção precoce de câncer ${ }^{7,16,20}$. As informações obtidas são analisadas do ponto de vista epidemiológico e constituem fonte de dados para o sistema de registro de morbidade.

Um "screening" realizado sistematicamente e pouco explorado no nosso meio é aquele realizado pelos bancos de sangue para o diagnóstico da infecção pelo Tripanossoma cruzi, virus da hepatite B, $T$. pallidum, e mais recentemente pelo vírus da Imunodeficiência Humana ${ }^{28}$. As informações colhidas adquirem importância epidemiológica por representarem parcela significativa da população adulta e por trazer dados que em estudos populacionais acarretariam grandes dificuldades operacionais, éticas, além de elevado custo ${ }^{12,26}$.

\footnotetext{
* Departamento de Saúde Coletiva do Instituto de Patologia Tropical e Saúde Pública da Universidade Federal de Goiás - Praça Universitária, $s / n$ - Setor Universitário - 74000 - Goiânia, GO - Brasil

* Banco de Sangue Goiano - Av. República do Líbano, 415 - qd. 56A - lote 9 - Setor Aeroporto - 74000 - Goiânia, GO - Brasil.

*** Instituto de Hemoterapia Goiano - Av. I.S.Carvalho, 623 - qd. 10A - lote 14 - Setor Aeroporto - 74000 - Goiânia, GO - Brasil. HEMOG - Av. Anhangüera, 5195 - Setor Campinas - 74000 - Goiânia, GO - Brașll.

*** HEMOLABOR Banco de Sangue - Rua 5-A, n: 90 - Setor Aeroporto - 74000 - Goiânia, GO - Brasil.
} 
O presente estudo pretende utilizar dados de "screening" sorológico realizado por bancos de sangue de Goiânia, para discutir o valor dos resultados como indicadores de morbidade e propor medidas para o aperfeiçoamento da coleta e análise das informações.

\section{MATERIAL E MÉTODO}

Foram analisados os dados referentes a 62.814 doações de sangue realizadas em dois bancos de sangue (BS) públicos e três privados, na cidade de Goiânia, Goiás, correspondendo ao total das doações realizadas no período de outubro de 1985 a outubro de 1987.

Os doadores eram, na sua maioria, procedentes de área urbana e familiares de pacientes internados nos hospitais da cidade. Somente $3 \%$ das doações eram provenientes de não-familiares. Por não haver remuneração pelo ato de doação, presume-se que não existam doadores profissionais. Cerca de $20 \%$ das doações foram realizàdas no setor público enquanto $80 \%$ foram realizadas nos BS particulares.

Nos BS, como procedimento de rotina, são excluídos como doadores os indivíduos com idade inferior a 18 anos, com relação peso/altura inadequada, os hipertensos e indivíduos com história de icterícia, doença de Chagas ou malária. Após o exame clínico e anamnese o sangue é coletado e testado sendo classificado como reagente ou não para fins de utilização.

Para a doença de Chagas são realizadas duas técnicas simultaneamente: a fixação de complemento (FC) e a hemaglutinação (HA) - um dos bancos de sangue público realiza somente a HA. Em caso de resultados discordantes, considera-se positivo os exames reagentes a uma terceira técnica (reação de imunofluorescência). Para sífilis utiliza-se o VDRL qualitativo em todos os BS, sendo que em dois bancos uma reação adicional de FC é utilizada. A hepatite B é investigada pela $\mathrm{HA}$ nos $\mathrm{BS}$ públicos e pela radioimunoensaio (RIE) nos privados. A detecção de infecção pelo vírus da AIDS pela enzima-imuno-ensaio (ELISA) teve início de forma rotineira, em outubro de 1985 , nos bancos de sangue privados, e, em março de 1987, nos públicos.

Todos os exames sorológicos dos BS privados, com exceção do teste de ELISA, são realizados por uma central de laboratório. Este fato permite maior comparabilidade entre os resultados obtidos no setor privado. Os BS públicos realizam os exames em seus próprios laboratórios.

Foram calculadas taxas de soroprevalência para cada doença e o valor preditivo negativo
(VPN) do interrogatório para doença de Chagas e hepatite B. O VPN foi calculado como a razão entre o número de resultados sorológicos negativos e o total de indivíduos que negaram a doença na anamnese. $O$ valor preditivo positivo (VPP) do interrogatório, ou seja, a probalidade de se obter um exame sorológico positivo nos indivíduos que afirmaram passado de doença na anamnese, não pode ser calculado devido ao não cadastramento desses candidatos quando excluídos pelo interrogatório. Como não foi possivel computar o número de indivíduos que doaram sangue mais de uma vez no período de estudo, todos os cálculos foram efetuados usando o total de doações de sangue registradas como denominador.

Por tratar-se de um estudo retrospectivo não foi possível uma melhor caracterização da população de doadores para o cálculo de coeficientes padronizados, para comparação com a população geral.

As diferenças entre as prevalências encontradas foram analisadas estatisticamente através do teste de Chi-quadrado. O risco relativo associado à concomitância de soropositividade (mais de um teste sorológico positivo) foi estimado através do cálculo de "Odds ratio" e apresentado com limites de confiança de $95 \%$ pelo método de Woolf.

\section{RESULTADOS}

A soroprevalência calculada para a doença de Chagas foi bastante semelhante em todos os bancos de sangue independentemente da técnica utilizada $(3,1 \%$ a $3,8 \%$ ) (Tabela 1). Para hepatite $B$ a prevalência variou de $0,7 \%$ a $1,8 \%$, diferença não significante estatisticamente $(p>0,05)$. Com relação a AIDS encontrou-se uma prevalência de $0,2 \%$ no banco de sangue $\mathbf{B}$, valor superior às demais taxas observadas $(0,0 \%$ a $0,1 \%)$, embora não estatisticamente significante $(p=0,56)$. Resultados discrepantes entre os bancos de sangue públicos e privados foram obtidos com o VDRL. Enquanto nos bancos privados a soroprevalência para sífilis situou-se em torno de $4,4 \%$, nos públicos ela foi de $0,8 \%$ e $5,1 \%$, respectivamente $(p<0,05)$.

A Tabela 2 compara a soroprevalência obtida pelas diferentes técnicas para cada doença. Embora tenham sido observadas diferenças estatisticamente significantes entre as técnicas, não se pode concluir se as diferenças seriam explicadas por diferente sensibilidade dos métodos empregados, por diferenças populacionais, ou ainda, por diferenças entre os laboratórios.

$O$ valor preditivo negativo do interrogatório foi de $96,9 \%$ e $96,7 \%$ para doença de Chagas e 
$98,7 \%$ e $99,2 \%$ para hepatite $\mathrm{B}$, dependendo da técnica considerada. Isto significa que em cada 100 indivíduos que negaram passado de doença de Chagas ou icterícia, três, aproximadamente, apresentaram resultados sorológicos positivos para doença de Chagas, e um para hepatite (Tabela 3).

A análise da concomitância de soropositividade, entre as diferentes doenças, não mostrou

\section{TABELA 1}

Soroprevalência para Doença de Chagas, Sifilis, Hepatite B e AIDS em bancos de sangue de Goiânia, segundo método empregado, outubro 1985 a outubro 1987.

\begin{tabular}{|c|c|c|c|c|c|c|c|c|}
\hline \multirow{3}{*}{$\begin{array}{c}\text { Banco } \\
\text { de } \\
\text { Sangue }\end{array}$} & \multicolumn{7}{|c|}{ Soroprevalência \% } & \multirow{3}{*}{$\begin{array}{c}\text { Total } \\
\text { Doaçōes }\end{array}$} \\
\hline & \multicolumn{2}{|c|}{ Chagas } & \multicolumn{2}{|c|}{ Sífilis } & \multicolumn{2}{|c|}{ Hepatite B } & \multirow{2}{*}{$\begin{array}{l}\text { AIDS } \\
\text { ELISA }\end{array}$} & \\
\hline & FC & HA & VDRL & $\mathrm{FC}$ & $\mathrm{HA}$ & RIE & & \\
\hline A & - & 3,85 & 0,83 & - & 0,77 & - & 0,00 & 6.206 \\
\hline B & 3,25 & $3,59^{*}$ & 5,08 & - & 1,80 & - & $0,21 * *$ & 6.548 \\
\hline $\mathrm{C}$ & 3,13 & 2,58 & 4,29 & 3,59 & - & 0,76 & 0,05 & $20: 195$ \\
\hline D & 3,90 & 3,68 & 4,30 & 4,22 & - & 0,72 & 0,03 & 11.930 \\
\hline $\mathbf{E}$ & 3,80 & 3,74 & 4,50 & - & - & 0,93 & 0,13 & 17.935 \\
\hline
\end{tabular}

A e B - bancos de sangue públicos

$C, D$ e E - bancos de sangue privados

* em 5.678 exames realizados

** em 1.845 exames realizados

- não realizado.

FC - fixação de complemento; HA - Hemaglutinação; VDRL - Venereal Diseases Research Laboratory (sorologia para sífilis); RIE - Radioimunoensaio; ELISA - Enzima-imuno-ensaio.

\section{TABELA 2}

Soroprevalência total para Doença de Chagas, Hepatite B, Sífilis e AIDS segundo método laboratorial utilizado em bancos de sangue de Goiânia, outubro 1985 a outubro 1987.

\begin{tabular}{|c|c|c|c|c|c|}
\hline \multirow{2}{*}{ Doença } & \multirow{2}{*}{ Método } & \multicolumn{2}{|c|}{ Exs. Positivos } & \multirow{2}{*}{$\begin{array}{c}\text { Total } \\
\text { Exames }\end{array}$} & \multirow{2}{*}{$\begin{array}{l}\text { Significância } \\
\text { Estatística }\end{array}$} \\
\hline & & $n$ & $\%$ & & \\
\hline Chagas & $\begin{array}{l}\mathrm{FC} \\
\mathrm{HA}\end{array}$ & $\begin{array}{l}1726 \\
2077\end{array}$ & $\begin{array}{l}3,04 \\
3,30\end{array}$ & $\begin{array}{l}56608 \\
62814\end{array}$ & $p=0,01$ \\
\hline Sífilis & $\begin{array}{l}\text { VDRL } \\
\text { FC }\end{array}$ & $\begin{array}{l}2568 \\
1231\end{array}$ & $\begin{array}{l}4,11 \\
3,83\end{array}$ & $\begin{array}{l}62814 \\
32125\end{array}$ & $p=0,05$ \\
\hline Hepatite B & $\begin{array}{l}\text { HA } \\
\text { RIE }\end{array}$ & $\begin{array}{l}168 \\
409\end{array}$ & $\begin{array}{l}1,31 \\
0,81\end{array}$ & $\begin{array}{l}12754 \\
50060\end{array}$ & $p=0,01$ \\
\hline AIDS & ELISA & 44 & 0,07 & 58111 & \\
\hline
\end{tabular}

FC - Fixação de complemento; HA - Hemaglutinação; VDRL - Venereal Diseases Research Laboratory (sorologia para sífilis); RIE - Radioimunoensaio;ELISA - enzima-imuno-ensaio

\section{TABELA 3}

Valor preditivo negativo do interrogatório para Hepatite B e Doença de Chagas em bancos de sangue de Goiânia.

\begin{tabular}{lccccc}
\hline Doença & Técnica & Positivos & Negativos & Total & VPN $\%$ \\
\hline \multirow{2}{*}{ Hepatite B } & HA & 168 & 12586 & 12754 & 98,7 \\
& RIE & 409 & 49651 & 50060 & 99,2 \\
Doença de Chagas & FC & 1726 & 54882 & 56608 & 96,9 \\
& HA & 2077 & 60737 & 62814 & 96,7 \\
\hline
\end{tabular}

VPN - valor preditivo negativo; HA - hemagiutinaçāo, RIE - radioimunoensaio; FC - fixaçāo de complemento. 
risco maior nos indivíduos com um exame positivo. A associação hepatite $\mathrm{B}$ e Chagas apresentou um risco relativo de 1,0 com limite de confiança $(95 \%)$ de 0,2 a 4,3 , e a associação sífilis e hepatite $B$, um risco relativo de 0,9 com limites de confiança de 0,2 a 3,8 , portanto ambos os limites incluindo a unidade.

\section{DISCUSSÃO}

A validade de dados epidemiológicos como fonte de informação de morbidade depende da abrangência do método de coleta e da representatividade da amostra em termos populacionais.

No entanto, ao se analisar dados de "screening" sorológico em banco de sangue como fonte de informação de morbidade deve-se considerar que os doadores representam amostra bastante selecionada. Ela é constituída basicamente por indivíduos adultos do sexo masculino e previamente auto selecionada do ponto de vista de morbidade.

Nos países em desenvolvimento, os sistemas de registro de morbidade e notificação oficial, mesmo quando implantados de forma sistemática, são deficientes em termos de cobertura podendo não ser representativos da população ge$\mathrm{ral}^{25}$. Em contrapartida, a triagem sorológica nos bancos de sangue é realizada de forma rotineira, cobrindo parcela significativa da população, que pelo caráter voluntário pode incluir grupos de risco para as diferentes doenças. A exploração desses dados como fonte de informação epidemiológica não apresenta os problemas operacionais e éticos dos inquéritos populacionais.

A existência de um registro oficial de morbidade possibilitaria comparar e validar o resultado obtido nos bancos de sangue com a magnitude da doença na população. Entretanto, só em relação a AIDS isso se torna possível ${ }^{17}$. Considerando que o número de casos de AIDS notificados para o Estado de Goiás, até outubro de 1987, foi de $23^{2}$ e, aceitando uma subnotificação de cerca de $30 \%$ (estimativa do Ministério da Saúde) ${ }^{1}$, isto equivaleria a existência de um total de 32 casos. Baseando-se na relação de um doente para 50 a 100 infectados $^{8}$, estimar-se-ia um contingente de 1.600 a 3.200 indivíduos infectados na região. Paralelamente, se a soropositividade para o HIV, encontrada nas 58.111 doaçōes $(0,07 \%)$, fosse projetada para a população maior de 15 anos, teríamos cerca de 1.900 infectados. Este valor situa-se, portanto, dentro dos limites esperados pela notificação da doença, o que reforça a validade da triagem co- mo indicador de morbidade populacional. Além do mais, no caso da AIDS há ainda uma semelhança entre doadores e a população de maior incidência; são, na maioria, adultos do sexo masculino ${ }^{8}$.

Em relação a AIDS dois aspectos devem ser considerados para melhor utilização dos dados: a) o grande número de resultados falsos positivos em população de baixa prevalência ${ }^{29} \mathrm{com}$ a conseqüente diminuição da positividade pela repetição do teste de ELISA ${ }^{21,28}$; b) diferenças na proporção de grupos de risco entre a clientela dos bancos de sangue e a população em ge$\mathrm{ral}^{23}$.

No caso da hepatite B, os dados epidemiologicos mais citados são provenientes de bancos de sangue. Os estudos epidemiológicos são geralmente restritos aos grupos de risco ${ }^{6,22}$. Nas regioes norte e nordeste do Brasil, consideradas de alta endemicidade para a hepatite B $(8 \%$ a $20 \%)^{14}$, parece ocorrer contato precoce das crianças com o vírus (HBV) possivelmente por transmissão vertical $\mathrm{e} /$ ou intrafamiliar ${ }^{10}$. Talvez nessas regiōes onde a infecção é freqüente no período neonatal e na infância, os dados de bancos de sangue, que detectam a presença do HBsAg, não seriam o melhor espelho da prevalência em nível populacional. Em Goiás, trabalhos anteriores registraram uma prevalência para HBV de $0,9 \% 19$, sendo considerada entre baixa $(0,2 \%$ a $0,5 \%)$ e intermediária $(2 \%$ a $7 \%)$ endemicidade, conforme classificação de Zuckerman ${ }^{27}$. No presente estudo, os valores calculados de $0,8 \%$ a $1,3 \%$ (RIE e HA) são semelhantes aos registrados anteriormente. Como esse padrão epidemiológico sugere infecção pouco freqüente na infância ${ }^{10}$, os dados obtidos pelo rastreamento em população adulta poderiam ser usados como estimativa do número de infectados na população geral.

Com relação à doença de Chagas, existe o registro, em 1975, de uma soroprevalência de $10,43 \%$ em 4.372 doadores do Hospital das Clinicas de Goiânias, dados semelhantes aos da década anterior, também em doadores de sangue da mesma cidade $(11 \%)^{5}$. Na zona rural de Goiás, o inquérito sorológico nacional ${ }^{4}$ encontrou prevalência média de $7,4 \% \%^{4}$, menor portanto que os resultados obtidos em doadores de sangue no mesmo período. Os dados atuais de $3,0 \%$ e 3,3\% sugerem uma diminuição da prevalência da doença de Chagas entre candidatos a doadores quando comparada às últimas duas décadas, coincidindo com valores descritos para as maiores capitais brasileiras (3\% a $6 \%)^{13}$. Este decréscimo talvez possa ser explicado por uma diferente proporção de migrantes rurais nas áreas urbanas entre as décadas de 70 e $80^{\circ}$, 
ou talvez pela melhoria do controle da doença e redução da tranmissão, ocorrendo um "efeito coorte"; os doadores de 80 estariam menos infectados que os de 70 .

Para a sífilis a prevalência calculada de $4,1 \%$ poderia ser considerada superestimada devido a inespecificidade do VDRL por ser uma reação não treponêmica com possibilidade de resultados falso positivos ${ }^{11,24}$. Por este motivo e pela instabilidade do resultado do teste a validação da prevalência torna-se bastante difícil.

A análise sistemática dos dados de banco de sangue poderia, a nosso ver, representar importante fonte de informação sobre a morbidade populacional desde que adequadamente coletada e processada. Deve-se chamar atenção que cerca de $6 \%$ da população adulta da cidade de
Goiânia doou sangue durante os dois anos de estudo, o que constitui uma amostra importante para efeito de estudos epidemiológicos. Para 0 aperfeiçoamento desse sistema de informação seria fundamental que se incluísse no sistema de registro todos os candidatos a doação, mesmo aqueles excluidos pela anamnese, a fim de que também se possa calcular o VPP do interrogatório e obter melhor estimativa de prevalência. Outras características da clientela como procedência, profissão, nível sócio-econômico e frequêencia de retornos seriam também facilmente analisados com a implantação de sistema de registro centralizado, computadorizado, sob a coordenação das Secretarias Estaduais de Saúde.

ANDRADE, A.L.S.S. de et al. [Serological screening for infectious diseases in blood donors as morbidity indicator]. Rev. Saúde públ., S. Paulo, 23:20-5, 1989.

ABSTRACT: Screening tests of 62,814 blood donations carried out between October 85 and October 87 in all five blood banks of Goiania, Central Brazil, were analysed. The private institutions ( 3 blood banks) collected $80 \%$ of all donations and the remaining were collected by public blood banks. Seroprevalence of HIV, HBsAg, Chagas' disease and syphilis were compared with data obtained in the surveillance system and from previous surveys in an attempt to validate this source of health information. A seroprevalence of $0.07 \%$ for AIDS (one ELISA test) was calculated, which may suggest the presence of 1900 infected individuals, in the population of the State of Goias as a whole. This figure seems reasonably close to the official data obtained by compulsory notification. For Chagas' disease, hepatitis B and syphilis seroprevalence of $3.3 \%, 1.3 \%$ and $4.1 \%$ were obtained, respectively. The potential usefulness and the limitations of screening in blood banks as a source of realible morbidity indicator is discussed.

KEYWORDS: Blood banks. Mass screening. Serodiagnosis. Morbidity. Blood donors.

\section{REFERENCIAS BIBLIOGRÁFICAS}

1. AIDS BOLETIM EPIDEMIOLÓGICO. (Ministério da Saúde). Brasília, 1(1) 1987.

2. AIDS BOLETIM EPIDEMIOLOGICO. (Ministério da Saúde). Brasília, 1(3) 1987.

3. AZULAY, R.D.; RICART, J.C. de; MONTEIRO, C.A.A.; GRIPP, A.C.; RODRIGUES, P.C.; ZANON, V.; GARNEIRO, N,F. Sifilis: inquerito sorológico em diferentes grupos sócio-económicos na cidade de Niterói. Rev. Ass. med. bras., 25:85-6, 1979.

4. CAMARGO, M.E.; SILVA, G.R.; CASTILHO, E.A.; SILVEIRA, A.C. Inquérito sorológico da prevalencia de infecçăo chagásica no Brasil, 1975/1980. Rev. Inst. Med. trop. S.Paulo, 26:193-204, 1984.

5. CAMPOS, C.; REZENDE, J.; RASSI, A. Prevalência de Chagas no Banco de Sangue do Hospital das Clínicas de Goiânia. Possibilidade de falha de reaçăo de Machado Guerreiro na seleçăo de doadores. Rev. Soc. bras. Med. trop., 12:165-74, 1975.

6. CARRILHO, F.G.; BALDY, J.L. da; TAKATA, P.K.; ADUM, S.; ZETUNE, Z.M.R.P. Prevalence and study of assymptomatic carriers of hepatitis B surface antigen ( $\mathrm{HBsAg}$ ) in blood donors in Londrina, South of Brazil. GED: Gastroenteral. Endosc. dig., S. Paulo, 3(1):13-20, 1984.
7. CHAMBERLAIN, J. Failures of cervical cytology screening programme. Brit. med. J., 289:6449-50, 1984.

8. CURRAN, J.W.; MORGAN, W.M.; HARDY, A.M.; JAFFÉ, H.W.; DARROW, W.; DOWBLE, W.R. The epidemiology of AIDS: current status and future prospects. Science, 229:1352-7, 1985.

9. DIAS, J.C.P. Análise e perspectivas do controle da doença de Chagas no Brasil. Rev. bras. Malar., 35:109-19, 1983.

10. FAGAN, E. \& WILLIAN, R. Hepatitis caused by hepatitis B virus. The Practitioner, 231:371-8, 1987.

11. FELMAN, Y.M. \& NIKITA, J.A. Syphilis serology today. Arch. Dermatol., 116:84-9, 1980.

12. GEVERS, J.K.M. AIDS: screening of possible carriers and human rights. Hlth Pol., 7:13-9, 1987.

13. INFORMAÇÕES EPIDEMIOLOGICAS SUCAM. (Ministério da Saúde. SUCAM). Brasília, 4(39) 1986.

14. LYRA, L.G.; DAMASCENO, P.P.; COTRIM, P.; MOTA, E.; SILVA, L. Prevalence of antibody to hepatitis $B$ virus in an urban population on Northeast Brazil. Rev. Inst. Med. trop. S.Paulo, 28:406-9, 1986. 
15. MENDES, T.F.; KUT, H.; MEXAS, P.P.F.; HERBERT, B.A. Infection by hepatitis B virus in patients of a general hospital. Arq. Gastroenterol., 16:73-80, 1979.

16. MILLER, A.B. Screening for cancer: issues and future directions. J. chron. Dis, 39:1067-77, 1986.

17. MINISTÉRIO DA SAÚDE. Portaria número 542 , de 22 de dezembro de 1986. Diário Oficial da Uniao, Brasilia, 24 dez. 1986.

18. PROENÇA, N.G. \& FREITAS, T.H.P. A sifilis na atualidade, no Brasil. Rev. Ass. med. bras., 27:43.5, 1981.

19. ROSA, H.; CARNEIRO, O.; LEMOS, Z.P. Incidéncia do AgHBs em várias tribos de índios do Brasil Central. Rev. goiana Med., 22:91-3, 1976.

20. SHAPIRO, S.; STRAX, P.; VENET, L. Periodic breast cancer screening in reducing mortality from breast cancer. J. Amer. med. Ass., 215:1777-85, 1971.

21. SCHOOR, J.B.; BERKOWITZ, A.; CUMMING, P.D.; KATZ, A.J.; SANDLER, S.G. Prevalence of HTLV-III antibody in American blood donors. New Eng. J. Med., 313:384-5, 1985.

22. SILVA, L.C. da Hepatites agudas e crónicas. São Paulo, Sarvier, 1986.

23. SIVAK, S.L. \& WORMSER, G.P. How common is HTLV-III infection in the United States? New Engl. J. Med.., 313:1352, 1985.
24. TAKEDA, A.A.K.; NAKAMURA, P.M.; BARBOSA, S.F.C. Estudo comparativo entre as reaçoes de floculação, fixação de complemento, imunofluorescéncia indireta e microhemaglutinaçăo passiva para sifilis. Rev. Inst. Adolfo Lutz, 39:137-44, 1979.

25. ZICKER, F. \& ZICKER, E.M.S. Beneficios previdenciários por incapacidade como indicador de morbidade. Estudo da doença de Chagas em Goiás. Rev. goiana Med., 31:125-36, 1985.

26. ZUCKERMAN, A.J. Would screening prevent the international spread of AIDS? Lancet, 22:1208-9, 1986.

27. ZUCKERMAN, A.J. Controversies in immunization against hepatitis B. Hepatology, 5:1227-30, 1985.

28. WENDEL, S.N.; RUSSO, C.; BERTONI, R.R.; TSUNODA, N.M. AIDS and blood donors in Brazil. Lancet, 2:506, 1985.

29. WHITE, L.J. Acquired Immunodificiency Syndrome. Ann. intern. Med., 104:575-81, 1986.

Recebido para publicaçđo em 12/2/1988 Reapresentado em $3 / 11 / 1988$ Aprovado para publicaçđo em $4 / 11 / 1988$ 